\title{
Evaluación y mejoramiento de la calidad de la potencia eléctrica usando un nuevo enfoque teórico
}

\author{
Armando J. Ustariz-Farfan ${ }^{1 \S}$, Eduardo A. Cano-Plata ${ }^{2}$, Hernán E. Tacca ${ }^{3}$ \\ ${ }^{1 \S}$ Department of Electrical, Electronic and Computer Engineering, Universidad Nacional de Colombia, \\ Manizales, ajustarizf@unal.edu.co \\ ${ }^{2}$ Department of Electrical, Electronic and Computer Engineering, Universidad Nacional de Colombia, \\ Manizales,eacanopl@unal.edu.co \\ ${ }^{3}$ Department of Electronic Engineering, Universidad de Buenos Aires, Buenos Aires Argentina, \\ htacca@fi.uba.ar.
}

(Recibido: Noviembre 17 de 2010 - Aceptado: Mayo 18 de 2011)

\section{Resumen}

Las aplicaciones prácticas, tales como la incorporación de un nuevo enfoque teórico en la definición de estrategias de compensación instantánea y promediada para filtros activos de potencia en sistemas polifásicos y la descripción de un indicador global para la evaluación de la calidad de potencia, son introducidas en este artículo. Para alcanzar estas aplicaciones, una teoría generalizada de la potencia instantánea aplicada en sistemas no-sinusoidales, polifásicos y asimétricos es propuesta. El tensor de potencia instantánea se define en función del producto tensorial entre los vectores de tensión y corriente. Además, resultados simulados son incluidos.

Palabras clave: Compensador Activo de Potencia, Teoría de Potencia Instantánea, Sistemas Polifásicos, Producto tensorial

ELECTRICAL ENGINEERING

\section{Improvement and assessment of the electrical power quality using a new theoretical approach}

\begin{abstract}
Practical applications, such as the incorporation of the a new theoretical approach in the definition of the instantaneous and average compensation objectives for active power filters (APFs) in multiphase systems and the description of a global indicator for the evaluation of the power quality are introduced in this paper. In order to obtain these applications, a generalized theory of instantaneous power for nonsinusoidal unbalanced multiphase systems is proposed. The instantaneous power tensor is defined based on the tensor product between vectors of voltage and current. In addition, the simulated results are included.
\end{abstract}

Keywords: Active Power Compensator, Instantaneous Power Theory, Multiphase Systems, Tensor product 


\section{Introduction}

There is an ever increase of nonlinear loads connected to electrical systems, where the injection of harmonic currents cause distortion of the voltage waveform complicating the analysis of electromagnetic phenomena. The traditional power theory based on the concept of the average value can not fully describe the electrical phenomena under these conditions. Therefore, the difficulty of defining a theory that describes the power for nonsinusoidal, unbalanced and multiphase systems entices researchers around the world (Depenbrock et al, 2003; Czarnecki, 2004; Morsi \& El-Hawary, 2007; Leon \& Cohen, 2008).

On the other hand, the physical meaning of the components of the instantaneous and apparent power in electrical systems is a topic that has been studied for many years and is still being studied today. Considerations such as harmonic effects and the imbalance of electrical signals in the power components in multiphase systems are currently under discussion and have originated a great deal of theories.

Theories about the transfer of power in multiphase systems have emerged since last beginningcentury (Depenbrock, 1993). These theories show no constrains on the number of stages and the numbers of wires in the power systems. More recently, Willems, 1992 extends the instantaneous reactive power theory proposed by Akagi et al, 1984 to multiphase systems. In all these theories the authors propose the decomposition of the current in two orthogonal components. The first component is derived from the concept of instantaneous active power; while the second component is calculated as the complement to the previous component in order to obtain the total current. Therefore these theories do not present a formal definition of reactive power in multiphase systems.

Dai et al, 2004 proposed an alternative to define the concept of reactive power in multiphase systems which later is formalized (Salmerón \& Herrera, 2009). This new approach defines the instantaneous reactive power from the definition of the exterior product in terms of the tensor product. However, while in (Dai et al, 2004) a development for a system with the number of phase greater than three is not explicitly presented; in (Salmerón \& Herrera, 2009) there is no reciprocity relation between the mathematical operations introduced to define the components of the current and each term of power.

A generalized instantaneous power theory is proposed following this tensor approach. The approach proposed in this paper emphasizes the possibility to formally define reactive power in nonsinusoidal unbalanced multiphase systems. Additionally, this paper presents the definition of instantaneous power in the context of tensor algebra as a derivation of circuit components (conductances and susceptances). Section 2 introduces the concept of tensor product, which allows the definition of instantaneous power tensor applied to nonsinusoidal, unbalanced and multiphase systems. Section 3 presents a tensor approach of the instantaneous reactive theory. This approach allows the definition of instantaneous and average compensation objectives in n-phase systems. Section 4 describes an indicator based on the instantaneous power tensor to evaluate the power quality.

\section{Instantaneous power tensor theory}

The proposed method is based on the definition of an evolutionary expression of instantaneous power, called "instantaneous power tensor" (Ustariz, 2010), in order to geometrically interpret the dynamic behavior of electromagnetic phenomena, analogous to studies of deformation in the mechanics of solids.

Therefore, the goal proposed is to obtain a single expression containing the two components of the instantaneous power (active and reactive). Therefore, using the dyadic or tensor product (Itskov, 2007) the proposed expression is as follows:

$$
\wp_{i j}=\vec{v}_{i} \otimes \vec{i}_{j}=\vec{v} \vec{i}^{T}
$$


Where ${ }_{i j}$ is the instantaneous power tensor and subscript $T$ refers to the vector transpose. On the other hand, a multiphase electrical network was defined as one $n$-phase and $m$-wire power system related to the dimension of vector space, where voltage vector and current vector were defined assuming that, $R^{l}$ is the single-phase system, $R^{2}$ is the two-phase system with two-wire or three-wire, $R^{3}$ is the three-phase system with three-wire or four-wire and $R^{n}$ is the multiphase system with $n$ phase and $m$-wire.

Therefore, in a system of $n$-phase and $m$-wire (see Figure 1), where the instantaneous vectors of voltage and current are expressed as:

$$
\mathrm{k}=\left[\begin{array}{c}
v_{1} \\
v_{2} \\
\mathrm{M} \\
v_{n}
\end{array}\right], \quad \dot{L}=\left[\begin{array}{c}
i_{1} \\
i_{2} \\
\mathrm{M} \\
i_{n}
\end{array}\right]
$$

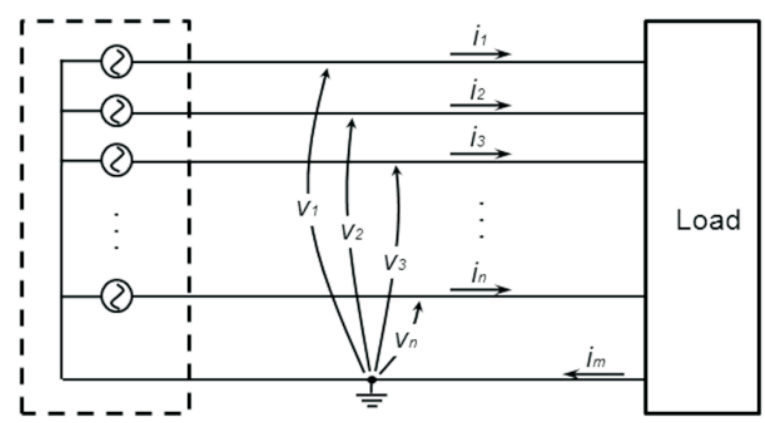

Figure 1: Multiphase system of n-phase and $m$-wire, $R^{n}$

Then, the instantaneous power tensor in $R^{n}$ is equal to:

$$
\wp_{i j}=\left[\begin{array}{c}
v_{1} \\
v_{2} \\
\mathrm{M} \\
v_{n}
\end{array}\right] \otimes\left[\begin{array}{c}
i_{1} \\
i_{2} \\
\mathrm{M} \\
i_{n}
\end{array}\right]=\left[\begin{array}{rrrr}
v_{1} i_{1} & v_{1} i_{2} & \mathrm{~L} & v_{1} i_{n} \\
v_{2} i_{1} & v_{2} i_{2} & \mathrm{~L} & v_{2} i_{n} \\
\mathrm{M} & \mathrm{M} & \mathrm{O} & \mathrm{M} \\
v_{n} i_{1} & v_{n} i_{2} & \mathrm{~L} & v_{n} i_{n}
\end{array}\right]
$$

Moreover, in a system of three-phase and threewire or four-wire, where the phases are identified by $a-b-c$, the instantaneous power tensor in $R^{3}$ is expressed as:

$$
\wp_{i j}=\left[\begin{array}{c}
v_{a} \\
v_{b} \\
v_{c}
\end{array}\right] \otimes\left[\begin{array}{l}
i_{a} \\
i_{b} \\
i_{c}
\end{array}\right]=\left[\begin{array}{lll}
v_{a} i_{a} & v_{a} i_{b} & v_{a} i_{c} \\
v_{b} i_{a} & v_{b} i_{b} & v_{b} i_{c} \\
v_{c} i_{a} & v_{c} i_{b} & v_{c} i_{c}
\end{array}\right]
$$

When $n=1$, it is the case of a single phase system, and the instantaneous values of voltage and current are scalars. Therefore, according to the traditional concept of instantaneous power in single phase systems the instantaneous power tensor in $R^{l}$ is equal to:

$$
\wp_{i j}=v i_{a} a
$$

\section{Tensor approach of the instantaneous reactive power}

\subsection{Orthogonal decomposition of the current vector}

According to Ec. (1):

$$
\wp_{i j}^{T}=\left(\vec{v} \vec{i}^{T}\right)^{T}=\vec{i} \vec{v}^{T}
$$

This expression implies that the current vector can be obtained from the definition of instantaneous power tensor as follows:

$$
\vec{i}=\frac{\wp_{i j}^{T}}{\vec{v} \cdot \vec{v}} v=\frac{\wp_{i j}^{T}}{\|\vec{v}\|^{2}} \vec{v}
$$

such that $\|\cdot\|$ denotes the Euclidean norm. Besides,

$$
\wp_{i j}^{T}=\wp_{i j}^{T}+\wp_{i j}-\wp_{i j}
$$

Now, replacing (7) in (8) the current vector can be expressed by,

$$
\vec{i}=\frac{\wp_{i j}^{T}+\wp_{i j}-\wp_{i j}}{\vec{v} \cdot \vec{v}} \vec{v}=\frac{\wp_{i j}}{\vec{v} \cdot \vec{v}} \vec{v}+\frac{\wp_{i j}^{T}-\wp_{i j}}{\vec{v} \cdot \vec{v}}=\vec{i}_{p}+\vec{i}_{q}
$$

Where, $\vec{i}_{p}$ is the instantaneous active current vector:

$$
\overrightarrow{i_{p}}=\frac{\wp_{i j}}{\vec{v} \cdot \vec{v}} \vec{v}=g_{i j} \vec{v}
$$

and $\vec{i}_{q}$ is the instantaneous reactive current vector:

$$
\vec{i}_{q}=\frac{\wp_{i j}^{T}-\wp_{i j}}{\vec{v} \cdot \vec{v}}=b_{i j} \vec{v}
$$

In Ec.(10) $g_{i j}$ is the instantaneous conductance tensor: 


$$
g_{i j}=\frac{\wp_{i j}}{\vec{v} \cdot \vec{v}}=\left[\begin{array}{cccc}
\frac{v_{1} i_{1}}{\|\vec{v}\|^{2}} & \frac{v_{1} i_{2}}{\|\vec{v}\|^{2}} & \mathrm{~L} & \frac{v_{1} i_{n}}{\|\vec{v}\|^{2}} \\
\frac{v_{2} i_{1}}{\|\vec{v}\|^{2}} & \frac{v_{2} i_{2}}{\|\vec{v}\|^{2}} & \mathrm{~L} & \frac{v_{2} i_{n}}{\|\vec{v}\|^{2}} \\
\mathrm{M} & \mathrm{M} & \mathrm{O} & \mathrm{M} \\
\frac{v_{n} i_{1}}{\|\vec{v}\|^{2}} & \frac{v_{n} i_{2}}{\|\vec{v}\|^{2}} & \mathrm{~L} & \frac{v_{n} i_{n}}{\|\vec{v}\|^{2}}
\end{array}\right]
$$

and in Ec. (11) $b_{i j}$ is the instantaneous susceptance tensor:

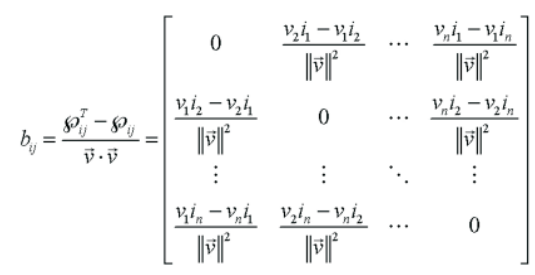

Moreover, the decomposition of the instantaneous current vector is characterized by:

$$
\begin{aligned}
\left\langle\vec{i}_{p} \cdot \vec{i}_{q}\right\rangle & =\vec{i}_{p}^{T} \vec{i}_{q}=\left(g_{i j} \vec{v}\right)^{T}\left(b_{i j} \vec{v}\right)=\vec{v}^{T} \frac{\vec{i}^{T}}{\vec{v}^{T} \vec{v}}\left(\frac{\vec{i}^{T}-\vec{v} \vec{i}^{T}}{\vec{v}^{T} \vec{v}}\right) \vec{v} \\
& =\frac{\left(\vec{v}^{T} \vec{i}\right)\left(\vec{v}^{T} \vec{i}\right) \vec{v}^{T} \vec{v}-\left(\vec{v}^{T} \vec{i}\right) \vec{v}^{T} \vec{v}\left(\vec{i}^{T} \vec{v}\right)}{\left(\vec{v}^{T} \vec{v}\right)^{2}} \\
& =\frac{\left(\vec{v}^{T} \vec{i}\right)^{2}}{\left(\vec{v}^{T} \vec{v}\right)^{2}}\left(\vec{v}^{T} \vec{v}-\vec{v}^{T} \vec{v}\right)=0
\end{aligned}
$$

such that,

$$
\|\vec{i}\|^{2}=\left\|\vec{i}_{p}\right\|^{2}+\left\|\vec{i}_{q}\right\|^{2}
$$

\subsection{Orthogonal decomposition of the power tensor}

The decomposition of the instantaneous power tensor is obtained by applying the tensor product of the voltage vector on Ec. (9), thus:

$$
\vec{v} \otimes \vec{i}=\vec{v} \otimes\left(\vec{i}_{p}+\vec{i}_{q}\right)=\vec{v} \otimes \vec{i}_{p}+\vec{v} \otimes \vec{i}_{q}
$$

Here, the first term is the instantaneous active power tensor:

$$
{ }^{p} \wp_{i j}=\vec{v} \otimes \vec{i}_{p}=\vec{v} \otimes\left(g_{i j} \vec{v}\right)=\vec{v} \otimes\left(\vec{v} g_{i j}^{T}\right)=(\vec{v} \otimes \vec{v}) g_{i j}^{T}
$$

and the second term is the instantaneous reactive power tensor:

${ }^{q} \wp_{i j}=\vec{v} \otimes \overrightarrow{i_{q}}=\vec{v} \otimes\left(b_{i j} \vec{v}\right)=\vec{v} \otimes\left(\vec{v} b_{i j}^{T}\right)=(\vec{v} \otimes \vec{v}) b_{i j}^{T}$

Consequently,

$$
\bullet{ }_{i j} \bullet{ }^{p}{ }_{i j} \bullet{ }^{q} \bullet{ }_{i j}
$$

Moreover, Ec. (19) satisfies the orthogonality concept, in other words,

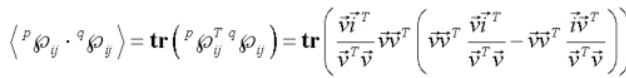

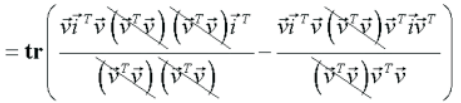

$$
\begin{aligned}
& =\operatorname{tr}\left(\vec{v}\left(\vec{i}^{T} \vec{v}\right) \vec{i}^{T}-\frac{\vec{v}\left(\vec{i}^{T} \vec{v}\right)\left(\vec{v}^{T} \vec{i}\right) \vec{v}^{T}}{\vec{v}^{2} \vec{v}}\right) \\
& =\vec{i}^{T} \vec{v}\left(\operatorname{tr}\left(\vec{v}^{T}\right)-\frac{\vec{v}^{T} \vec{i}}{\vec{v}^{T} \vec{v}} \operatorname{tr}\left(\vec{v}^{T}\right)\right) \\
& =\vec{i}^{T} \vec{v}\left(\vec{v}^{T} \vec{i}-\frac{\vec{v}^{T} \vec{i}}{\vec{v}} \vec{v} \vec{v} T \vec{v}\right)=0
\end{aligned}
$$

Here, the tr operator corresponds to the sum of the main diagonal elements (trace). Therefore, the decomposition of the instantaneous power tensor satisfies the following orthogonal relation:

$$
\left\|\wp_{i j}\right\|^{2}=\left\|{ }^{p} \wp_{i j}\right\|^{2}+\left\|{ }^{q} \wp_{i j}\right\|^{2}
$$

The importance of the new formulation is based on the fact that the expression for the instantaneous reactive power in multiphase systems is known, thanks to the decomposition of the tensor power, given in (19). Now, thanks to know the expression of instantaneous reactive power, there are other compensation strategies possible for the reference currents estimate for the control of active power filters, regardless of the number of phases of the power system (e.g. reactive power cancellation and distortion power cancellation).

The expression given in (21) is equivalent to the vector formula of the generalized instantaneous 
reactive power theory proposed by Willems 1992, where the instantaneous apparent power is defined as:

$$
s^{2}=p^{2}+q^{2}
$$

With $p$ defined as the instantaneous power transmitted to the load (instantaneous active power), thus:

$$
p=\vec{v} \cdot \vec{i}=v_{1} i_{1}+v_{2} i_{2}+\mathrm{L}+v_{n} \dot{i}
$$

and $q$ as the instantaneous imaginary power (instantaneous reactive power), determined by:

$$
q=\|\vec{v}\|\left\|\overrightarrow{i_{q}}\right\|=\|\vec{v}\|\left\|\vec{i}-\vec{i}_{p}\right\|
$$

Where,

$$
\vec{i}_{p}=\frac{p}{\|\vec{v}\|^{2}} \vec{v}
$$

Therefore, the following equivalences are established:

$$
s=\left\|\wp_{i j}\right\| ; \quad p=\left\|^{p} \wp_{i j}\right\| ; \quad q=\left\|{ }^{q} \wp_{i j}\right\|
$$

\section{Power quality improvement using instantaneous power tensor}

This section has a number of control strategies for active power filters (APFs), which can be implemented using the proposed tensor formulation. Table 1 shows a summary of the possible compensation objectives from the point of view of power in electrical systems (Montero et al, 2007; Salmerón \& Litran, 2010).
Given these possible compensation objective, the instantaneous vector of the referent current is defined as:

$$
\vec{i}_{(r e f)}=\frac{{ }^{p} \wp_{i j(r e f)}^{T}}{\vec{v} \cdot \vec{v}}+\frac{{ }^{q} \wp_{i j(r e f)}^{T}}{\vec{v} \cdot \vec{v}} \vec{v}
$$

Moreover, of the various options of the reference tensor shown in Table $1,{ }^{p}{ }^{\sim}{ }_{i j}$ is the variable term of the instantaneous active power tensor defined by:

$$
{ }^{p} \widetilde{\wp}_{i j}={ }^{p} \wp_{i j}-{ }^{p} \bar{\wp}_{i j}={ }^{p} \wp_{i j}-(\vec{v} \otimes \vec{v}) G_{i j}^{T}=(\vec{v} \otimes \vec{v})\left(g_{i j}^{T}-G_{i j}^{T}\right)
$$

Where $G_{i j}$ is the equivalent conductance tensor defined by:

$$
G_{i j}=\frac{\operatorname{tr}\left(\frac{1}{T} \int_{T}{ }^{p} \wp_{i j} d t\right)}{\vec{v} \cdot \vec{v}} \delta_{i j}
$$

With $\delta_{i j}$ defined as the "Kronecker's delta tensor" and used to construct the equivalent conductance tensor. While ${ }^{q} \sim_{i j}$ is the variable term of instantaneous reactive power, defined as:

${ }^{7} \tilde{\xi}_{g t}={ }^{q} \xi_{g}-{ }^{q} \bar{\xi}_{t}={ }^{q} \xi_{g}-(\vec{v} \otimes \vec{v}) B_{g}^{T}=(\vec{v} \otimes \vec{v})\left(b_{g}^{T}-B_{g}^{T}\right)$

where $B_{i j}$ is the equivalent susceptance tensor defined by:

$$
B_{i j}=\frac{\frac{1}{T} \int_{T}^{q} \wp_{i j} d t}{\vec{v} \cdot \vec{v}}
$$

\begin{tabular}{|c|c|c|}
\hline \multirow[b]{2}{*}{ Compensation objective-Power } & \multicolumn{2}{|c|}{ Reference Tensor } \\
\hline & ${ }^{P} 8 \mathrm{O}_{y\left(y, f^{\prime}\right)}$ & ${ }^{q} g_{i j(r e f)}$ \\
\hline Instantaneous reactive- $I R$ & 0 & ${ }^{q} \wp_{i j}$ \\
\hline Oscillating reactive- $O R$ & 0 & ${ }^{q} \tilde{\theta}_{v}$ \\
\hline Oscillating active and oscillating reactive $-O A / O R$ & $p \tilde{\wp}_{i j}$ & ${ }^{9} \tilde{\S}_{i j}$ \\
\hline Oscillating active and instantaneous reactive $-O A / R$ & ${ }^{p} \overrightarrow{8 O}_{y j}$ & ${ }^{Q} \wp_{y}$ \\
\hline
\end{tabular}

The block diagram of the control strategies for the determination of APF reference compensation currents is shown in Figure 2, where the compensation objectives selector block was implemented.

Table 1: Compensation Objectives in Electrical network 


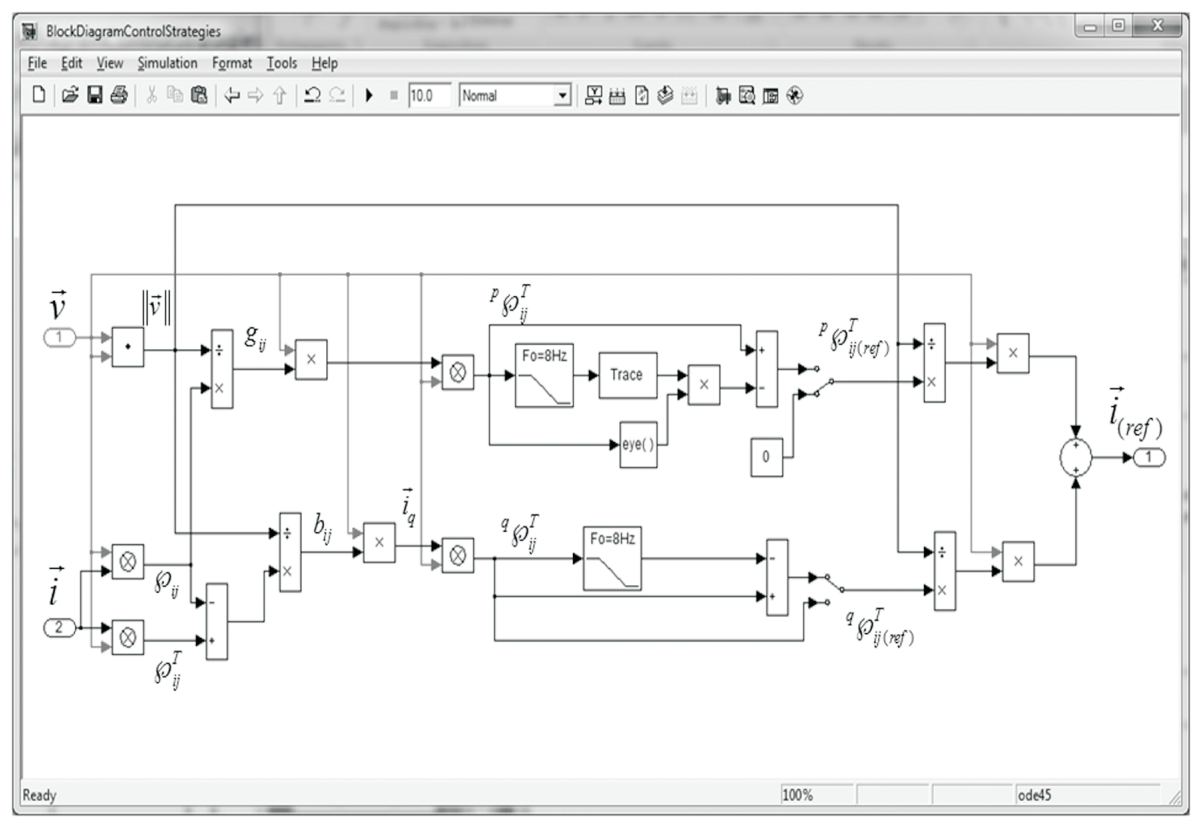

Figure 2: Block diagram of the control strategies for determination of APF reference current.

The different objectives of compensation have been applied to a nonlinear six-phase system. In this circuit, a sinusoidal and balanced three phase voltage source, feeds a triple wound transformer $\mathrm{Y}, \mathrm{Y} \bullet$. The secondary winding feeds a twelve pulse controller rectifier operating with a firing angle of $45^{\circ}$ with a source of current at the dc side assumed as load (Figure 3). This system was chosen because it is a practical case where six-phase waveforms can be identified.

The circuit shown in Figure 3 has been implemented in Matlab-Simulink. The active power filter is modeling as an ideal current source controlled by the referent current obtained with the proposed tensor formulation.

Figures 4 to 7 show the simulation results for the different compensation objectives. The voltage and current waveforms in these figures correspond to the first phase (phase-a in threephase side and phase-1 in six-phase side). In addition, $t=50 \mathrm{~ms}$ is the time in which the active filter is connected to the system. For all cases, the information is organized as follows.
Three-phase system (transformer primary side):

(a) Voltage waveform, $v_{a}$

(b) Current waveform, $i_{a \text {. }}$

(c) Active current waveform, $i_{p_{-} a}$

(d) Reactive current waveform, $i_{q_{a}}$

(e) Instantaneous active power, $\left\|{ }^{p} \wp_{i j}\right\|_{3 \varphi}$

(f) Instantaneous reactive power, $\left\|{ }^{q} \wp_{i j}\right\|_{3} \varphi$

Six-phase system (transformer secondary side):

(g) Voltage waveform, $v_{1}$

(h) Current waveform, $i_{1}$

(i) Active current waveform, $i_{p_{-} 1}$

(j) Reactive current waveform, $i_{q_{\perp} I}$

(k) Instantaneous active power, $\left\|^{p} \wp_{i j}\right\|_{6 \varphi}$

(1) Instantaneous reactive power, $\left\|{ }^{q} \wp_{i j}\right\|_{6 \varphi}$

The simulation results for the compensation objective $I R$ are shown in Figure 4 and summarized in the third column of Table 2. Here, the instantaneous reactive power is completely 


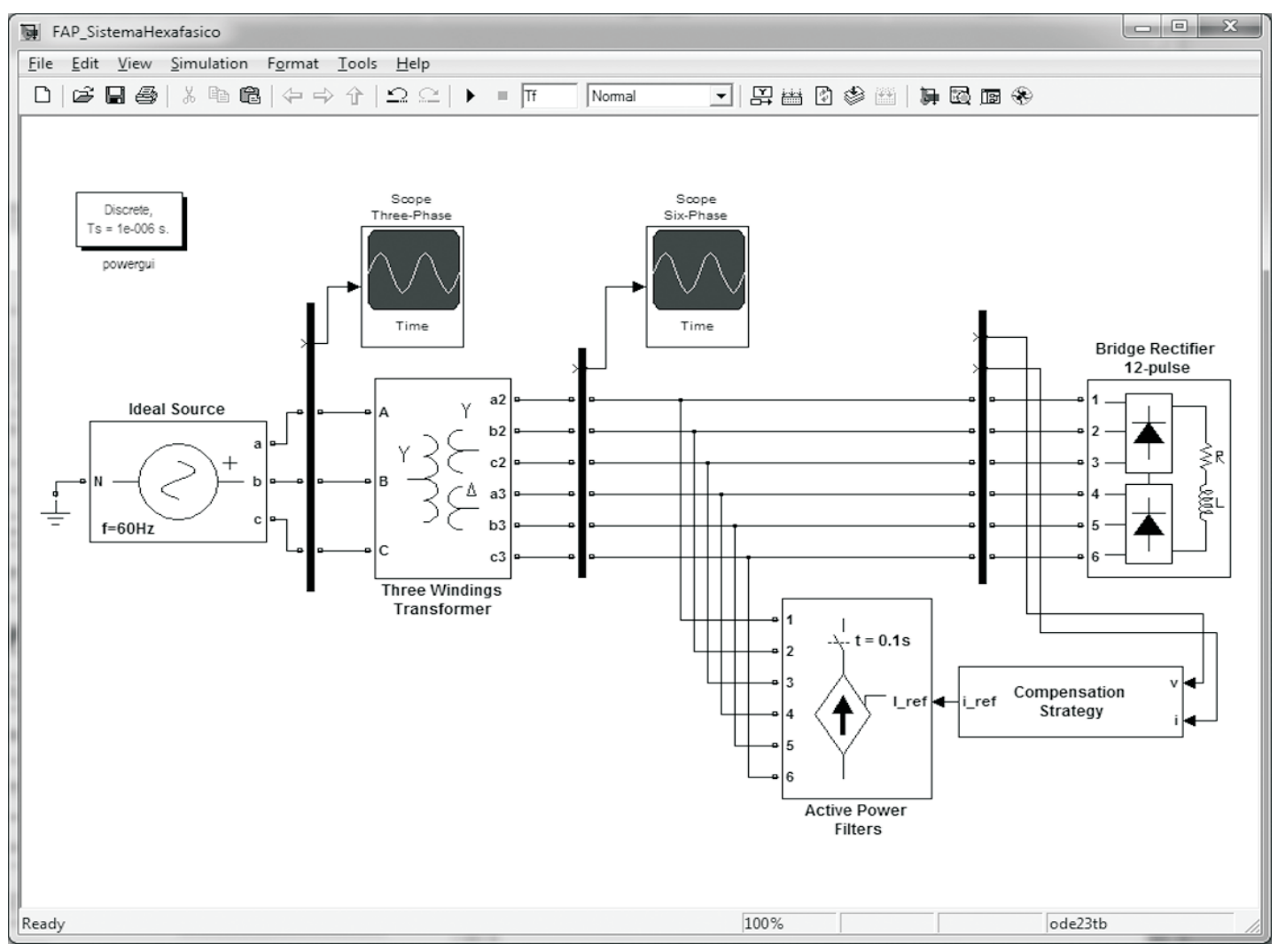

Figure 3.Three-phase to six-phase system compensated by a shunt APF implemented in MATLAB-Simulink

removed both on three-phase and six-phase side when the active filter is connected. Therefore, the instantaneous reactive power is fully compensated when the active filter is connected, while the delivered instantaneous active power equals the instantaneous active power consumed by the load. Regarding distortion, it's observed that the waveform of the current signals change but the total harmonic distortion not improvement.

The simulation results for the compensation objective $O R$ are shown in Figure 5 and summarized in the fourth column of Table 2. Now, when the active filter is connected, only the oscillating portion of the instantaneous reactive power is compensated thus, the source has to continue generating a constant instantaneous reactive power. The instantaneous active power delivered by the source remains equal to the instantaneous active power consumed by the load.
From the results shown in Figure 5, the instantaneous reactive current is delayed exactly by $90^{\circ}$ with the voltage both on threephase and six-phase side when the active filter is connected.

The simulation results for the compensation objective $O A / O R$ are shown in Figure 6 and summarized in the fifth column of Table 2. Here, the oscillating active and reactive powers are simultaneously compensated when the active filter is connected. This time, the waveform of the current is sinusoidal but still keeps the phase shift obtained in the compensation objective $O R$. From the results shown in Figure 6, the instantaneous active current is perfectly in phase with the voltage and instantaneous reactive current is delayed exactly by $90^{\circ}$ with the voltage both on three-phase and six-phase side when the active filter is connected. 

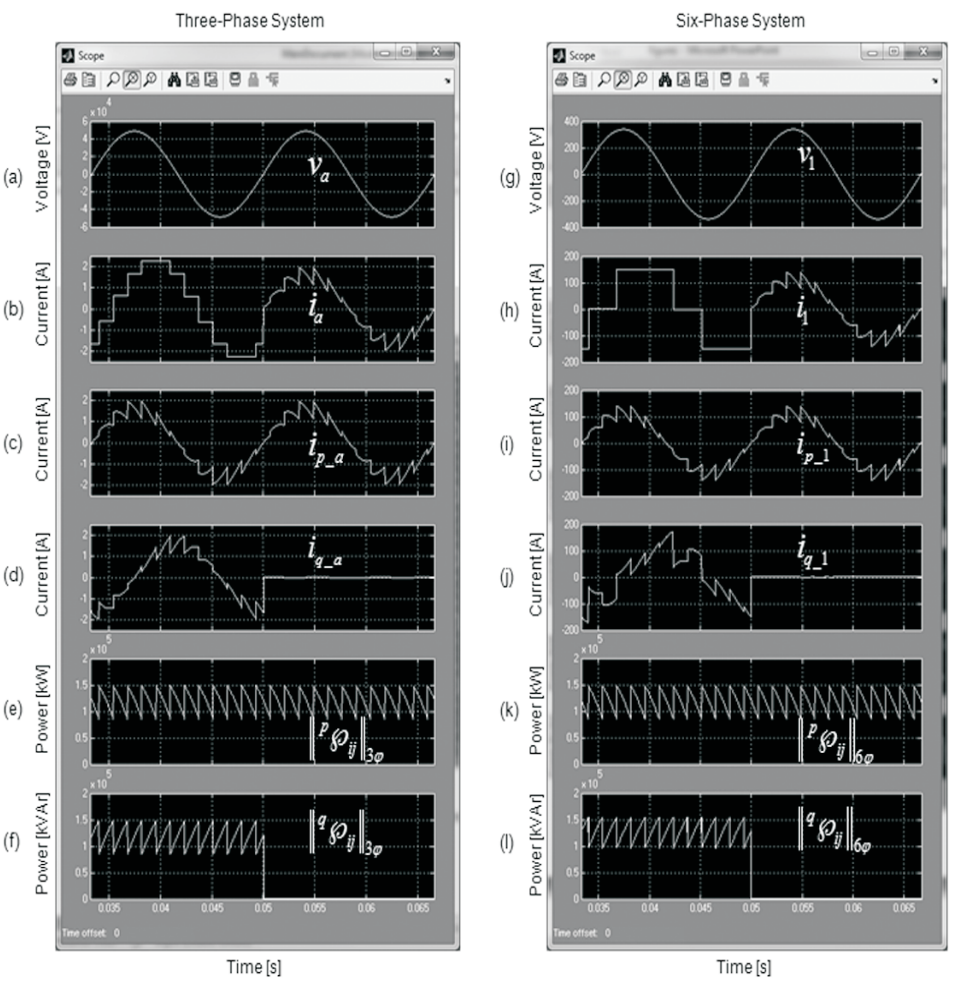

Figure 4.Simulation results: compensation objective IR
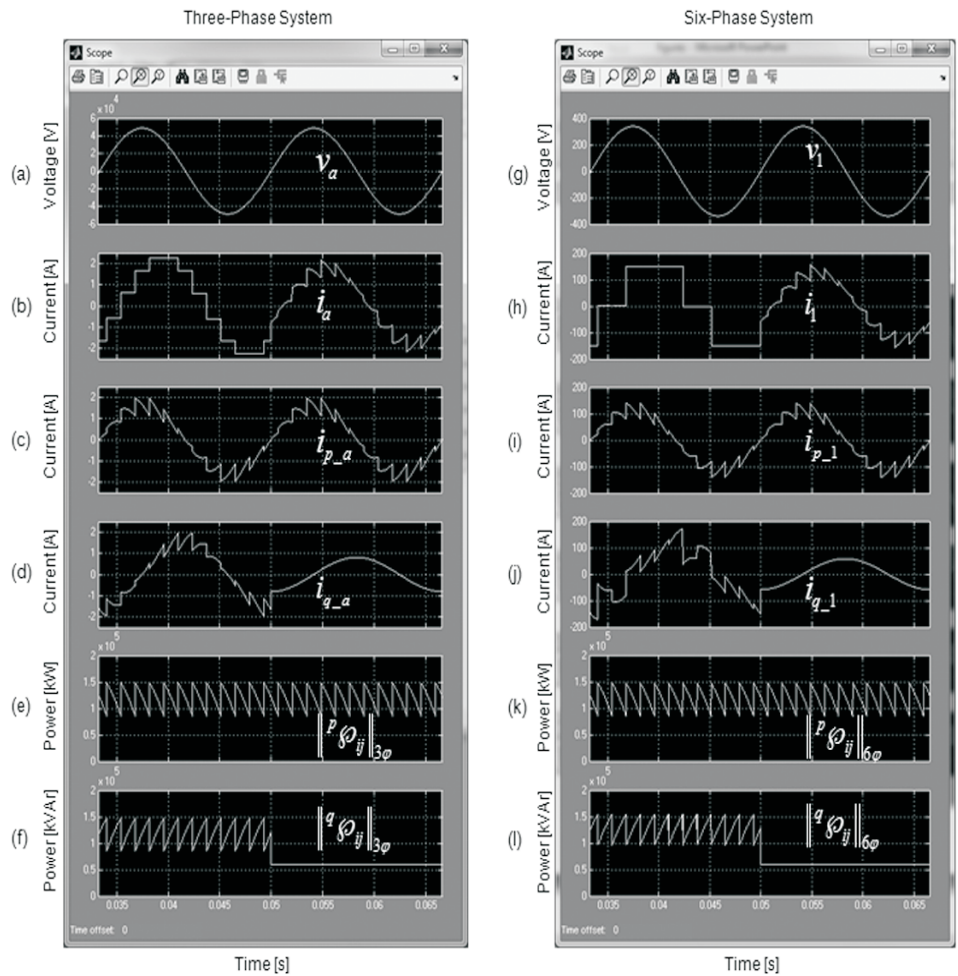

Figure 5.Simulation results: compensation objective OR 

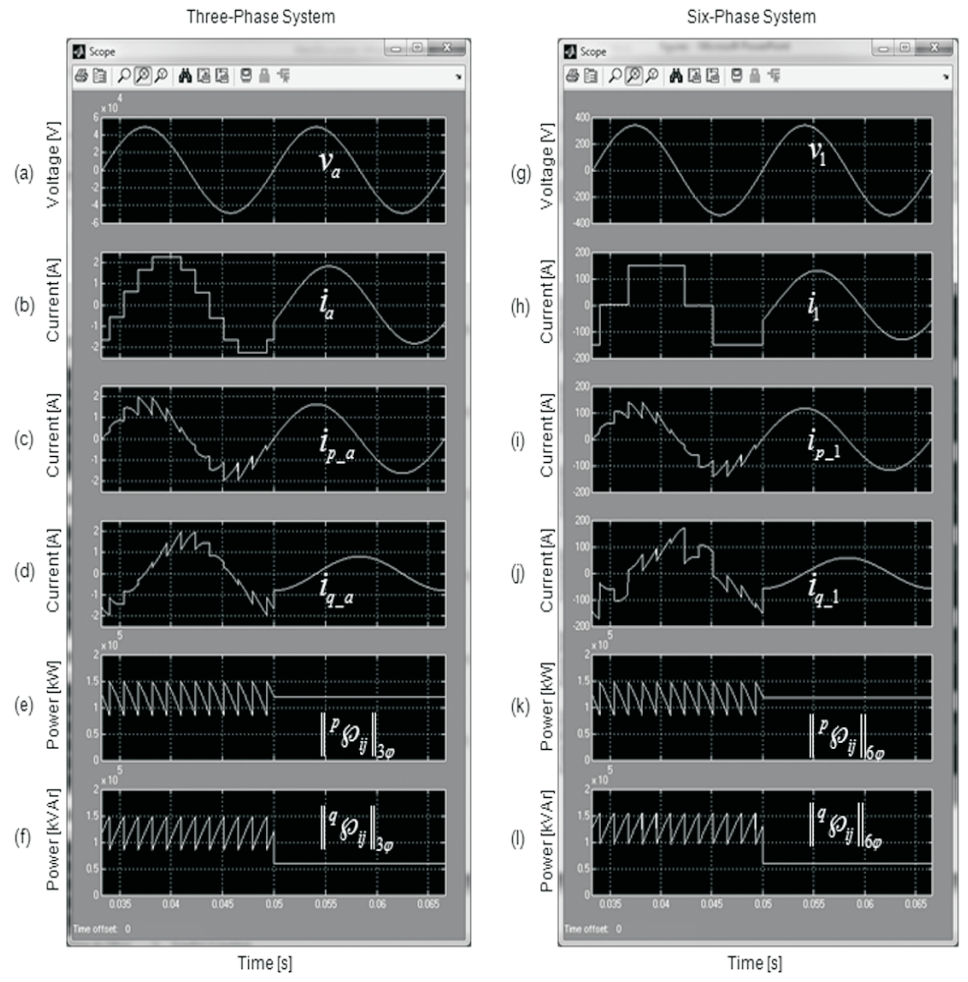

Figure 6.Simulation results: compensation objective OA/OR
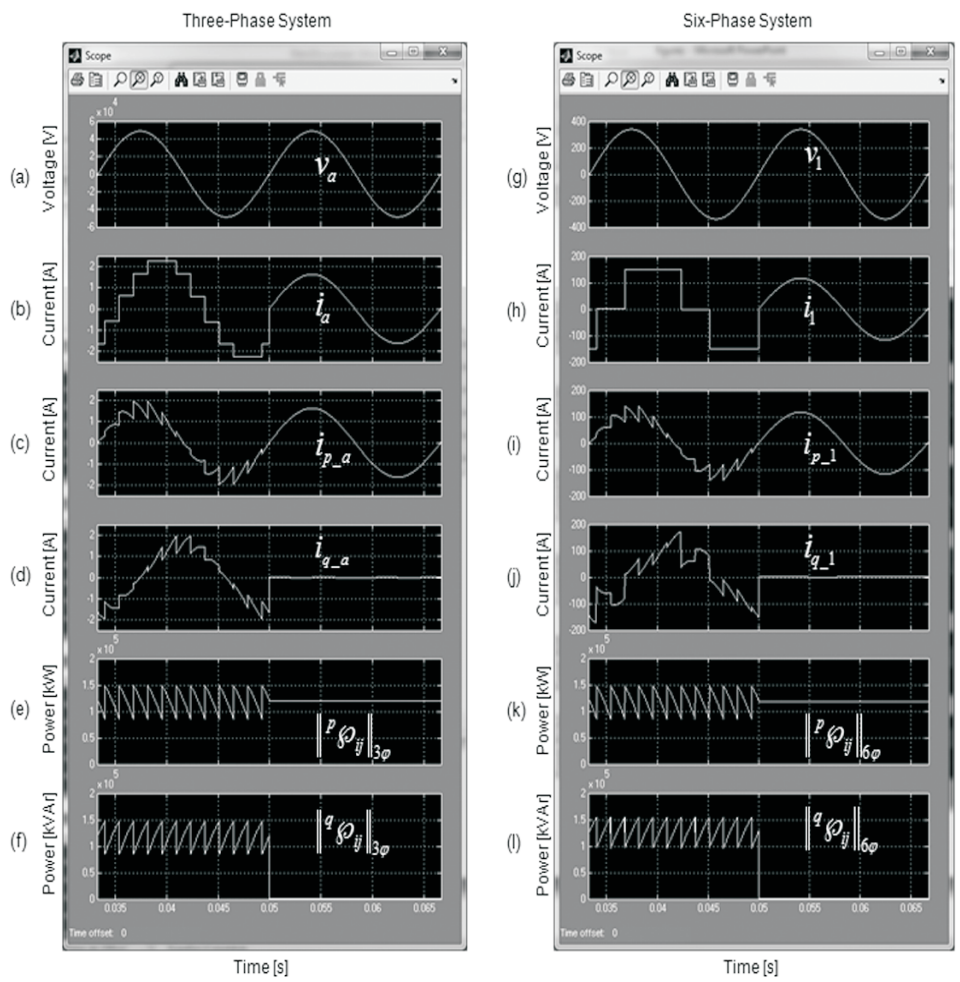

Figure 7.Simulation results: compensation objective OA/IR 
Table 2: Summary of the Simulation Results

\begin{tabular}{|c|c|c|c|c|c|}
\hline Factor & $i_{\text {load }}$ & $i_{p(I R)}$ & $i_{p(O R)}$ & $i_{p(O A / O R)}$ & $i_{p(O A / I R)}$ \\
\hline$I_{r m s}[A]$ & 1.650 & 1.169 & 1.303 & 1.291 & 1.156 \\
\hline$I_{f}[A]$ & 1.631 & 1.156 & 1.291 & 1.291 & 1.156 \\
\hline$T H D_{I}[\%]$ & 15.20 & 15.18 & 13.59 & 0.000 & 0.000 \\
\hline$d P F$ & 0.710 & 1.000 & 0.895 & 0.896 & 1.000 \\
\hline$P[k W]$ & 119.8 & 119.8 & 119.8 & 119.8 & 119.8 \\
\hline$S_{e}[k V A]$ & 170.8 & 121.0 & 134.9 & 133.6 & 119.8 \\
\hline$P F$ & 0.702 & 0.989 & 0.887 & 0.896 & 1.000 \\
\hline
\end{tabular}

The simulation results for the compensation objective $O A / I R$ are shown in Figure 7 and summarized in the sixth column of Table 2. Now, the oscillating active power and the instantaneous reactive power are compensated when the active filter is connected. In this compensation objective the waveform of the current signal is also sinusoidal. In addition the voltage and current signals are in phase.

From the results shown in Table 2, as expected, the only strategy which gets to compensate the combined effect is the correspondent to the compensation objective $O A / I R$. The result of this strategy is the closer to the ideal system.

The terms relating to power concepts in the Table 2 are based on the new definitions of power, proposed by the IEEE Working Group on Nonsinusoidal Situations as collected in the IEEE Standard 1459-2000 (Emanuel, 2004).

\section{Power quality assessment using instantaneous power tensor}

\subsection{Conventional indices for power quality assessment}

The first step toward power quality assessment is the definition of power quality indices able to quantify the deviation from an ideal reference situation and quantify the detrimental effects of this deviation (Cristaldi et al, 2002; Dell'Aquila et al, 2004; Lin \& Domijan, 2005; Shin et al, 2006; Ferrero, 2008). Traditionally, in stationary state the power quality assessment is based on individual indicators such as total harmonic distortion $T H D_{\mathrm{I}}$, load current unbalance factor $U F$ and reactive factor $Q F$. Also, the maximum value between these indices is chosen as a global indicator of power quality $G I$, thus:

$$
\begin{gathered}
T H D_{I}=\max \left(\frac{\sqrt{\sum_{h(a)}^{2}}}{I_{1(a)}}, \frac{\sqrt{\sum_{h(b)}^{2}}}{I_{1(b)}}, \frac{\sqrt{\sum I_{h(c)}^{2}}}{I_{1(c)}}\right) \\
U F=\frac{-I_{1}}{{ }^{+} I_{1}} \\
Q F=\frac{Q_{1(a)}+Q_{1(b)}+Q_{1(c)}}{P_{a}+P_{b}+P_{c}} \\
G I=\max \left(T H D_{I}, U F, Q F\right)
\end{gathered}
$$

where, the $I_{h(k)}$ is the current harmonic in the phase$k(k=a, b, c),{ }_{I}{ }_{l}$ and ${ }^{+} I_{l}$ are the fundamental negative and positive sequence components and $Q_{l(k)}$ is the fundamental reactive power of phase- $k$.

\subsection{Symmetrical and anti-symmetrical decomposition}

Section III introduces a decomposition of the instantaneous power tensor focused on the division of the instantaneous current vector into two orthogonal components: the first component in phase with the voltage vector, which is related to the instantaneous active power and the other 
component related to the instantaneous reactive power. This section defines another tensor decomposition, taking into account that any second order tensor can be unequivocally expressed as the sum of a symmetric tensor and an anti-symmetric thus:

$$
\wp_{i j}=\frac{1}{2}\left[\wp_{i j}+\wp_{i j}^{T}\right]+\frac{1}{2}\left[\wp_{i j}-\wp_{i j}^{T}\right]
$$

This expression can be written in compact form, as:

$$
\wp_{i j}={ }^{s y m} \wp_{i j}+{ }^{a n t} \wp_{i j}
$$

The operation leading from an arbitrary tensor with components $\bullet_{i j}$ to the tensor with components $\bullet{ }_{i j} \bullet{ }_{i j}^{T}$ is called symmetrization, while the operation leading to the tensor with components

$\bullet{ }_{i j} \bullet{ }_{i j}^{T}$ is called anti-symmetrization.

The concept of symmetrization in electrical systems corresponding to the definition of symmetrical or ideal circuits is introduced, in the next item, using this decomposition.

\subsection{Deviation factor of power quality based on the tensor analysis}

Now, a single discriminating factor to power quality assessment is proposed based on the symmetrical and anti-symmetrical decomposition of instantaneous power tensor. This factor afterwards could be used to penalize or to compensate the customers at the point of common coupling (PCC), similarly as the power factor has been used.

Establishing this factor implies to define the ideal power system as one circuit composed of a balanced and sinusoidal voltage source (reference source) supplying a resistive balanced load (reference load). Any situation producing nonconformity with respect to these ideal conditions supposes a quality loss. Moreover, this ideal circuit is the unique power system that presents a totally symmetric instantaneous power tensor.

The evaluation of these nonconformities can be quantified through the definition of a new power quality index, named instantaneous deviation indicator of power quality $I D I_{p q}$, stated by

$$
I D I_{p q}=\sqrt{\frac{\sum_{i=1}^{n} \sum_{j=1}^{n}\left(\wp_{i j}-{ }^{\text {ideal }} \wp_{i j}\right)^{2}}{\sum_{i=1}^{n} \sum_{j=1}^{n} \text { ideal } \wp_{i j}^{2}}}
$$

and next, the deviation factor of power quality defined as:

$$
D F_{p q}=\sqrt{\frac{1}{T} \int_{T}\left(I D I_{p q}\right)^{2} d t}
$$

In the Eq (38) the term ${ }^{\text {ideal }} \wp_{i j}$ corresponds to the instantaneous power tensor of the ideal power system (tensor totally symmetric). The ideal instantaneous power tensor is calculated as follows:

$$
{ }_{\text {ideal }} \wp_{i j}=\vec{v}_{f}^{+} \otimes \vec{i}_{f(a c t i v)}^{+}=\vec{v}_{f}^{+} \otimes \frac{\operatorname{tr}\left(\vec{\wp}_{i j}\right)}{\frac{1}{T} \int_{T}\left(\vec{v}_{f}^{+} \vec{v}_{f}^{+}\right)} \vec{v}_{f}^{+}=\left(\vec{v}_{f}^{+} \otimes \vec{v}_{f}^{+}\right) G_{(\text {ideal })}
$$

where $v_{f}^{+}$is the voltage vector with a single fundamental positive-sequence component and $G_{\text {(ideal) }}$ is the equivalent conductance of the ideal power system.

In this paper, the power quality assessment approach proposed based on the instantaneous power tensor theory has been applied to a threephase, four-wire system. The analyzed electrical network is similar to the one presented in Figure 8. The electrical network was simulated in ATPEMTP. In addition, the $D F_{p q}$ index is in contrasted against the GI index. For comparison proposes, 
several simulations done using both ideal and distorted voltage under different load current conditions. The details of each load connected at the PCC are shown in Figure 9.

The RMS values of the voltage and the values of the parameters of the power system under study are shown in Table 3. The system impedance is assumed to be of a resistance of $0.892 \mathrm{~m} \bullet$ in series with an inductance of $0.016 \mathrm{mH}$.
Table 4 shows a summary of the simulated cases. In the Table 4, the script "on" refers to the connection of the source or disturbing load in the PCC, while the script "off" refers to the nonconnection. Figure 10 shows the voltage and current waveforms measurement in the case $\mathrm{H}$.

Table 5 shows the values of the individual conventional indicators used in the evaluation of the power quality in each of the simulated cases.

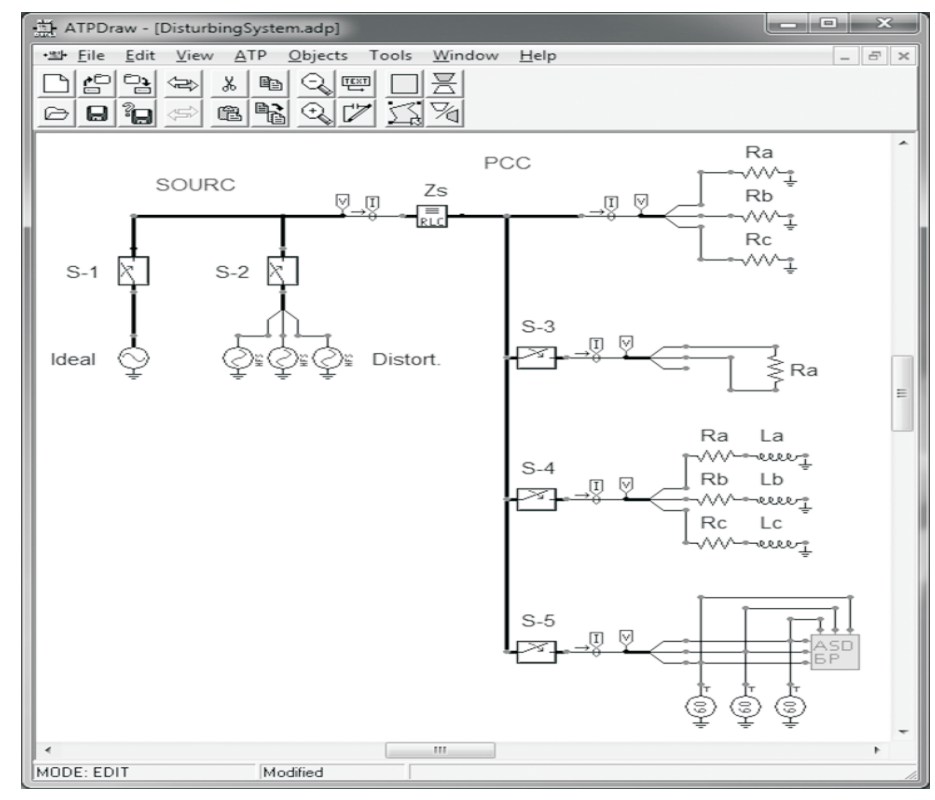

Figure 8.The studied example implemented in ATP-EMTP

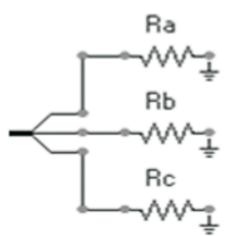

(a)

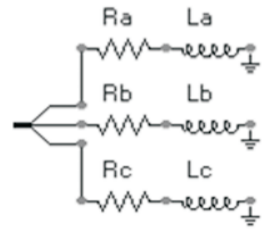

(c)

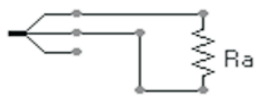

(b)

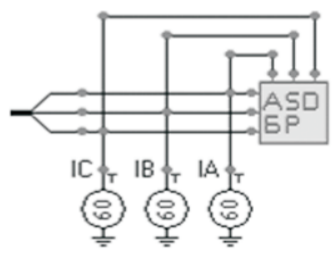

(d)

Figure 9.Three-phase loads connected in the studied example: (a) ideal load, (b) disturbing load \#1(unbalanced), (c) disturbing load \#2 (inductive) and (d) disturbing load \#3 (nonlinear). 
Table 3: System Parameters

\begin{tabular}{|c|c|c|c|c|}
\hline \multirow[t]{2}{*}{ Supply } & \multirow[t]{2}{*}{ Ideal } & \multicolumn{3}{|c|}{ Distorted } \\
\hline & & \multicolumn{2}{|c|}{ Fundamental } & $3^{\text {rd }}$ harmonic \\
\hline$V_{a}\left[\mathrm{~V}_{\mathrm{rms}}\right]$ & $220 \angle 0^{\circ}$ & \multicolumn{2}{|c|}{$201 \angle 0^{\circ}$} & $35.07 \angle 0^{\circ}$ \\
\hline$V_{b}\left[\mathrm{~V}_{\mathrm{rms}}\right]$ & $220 \angle-120^{\circ}$ & \multicolumn{2}{|c|}{$220 \angle-120^{\circ}$} & $46.21 \angle 0^{\circ}$ \\
\hline$V_{c}\left[\mathrm{~V}_{\mathrm{rms}}\right]$ & $220 \angle-240^{\circ}$ & \multicolumn{2}{|c|}{$220 \angle-240^{\circ}$} & $46.21 \angle 0^{\circ}$ \\
\hline \multirow[t]{2}{*}{ Load } & \multirow[t]{2}{*}{ Ideal } & \multicolumn{3}{|c|}{ Disturbing } \\
\hline & & $\# 1$ & $\# 2$ & $\# \mathbf{3}$ \\
\hline$R_{a}[\Omega]$ & 1.32 & 2.67 & 1.17 & - \\
\hline$R_{b}[\Omega]$ & 1.32 & - & 1.17 & - \\
\hline$R_{c}[\Omega]$ & 1.32 & - & 1.17 & - \\
\hline$L_{a}[\mathrm{mH}]$ & - & - & 2.53 & - \\
\hline$L_{b}[\mathrm{mH}]$ & - & - & 2.53 & - \\
\hline$L_{c}[\mathrm{mH}]$ & - & - & 2.53 & - \\
\hline$I_{a}$ (fundamental) $\left[\mathrm{A}_{\mathrm{rms}}\right]$ & - & - & - & $107 \angle 51^{\circ}$ \\
\hline$I_{a}\left(5^{r d}\right.$ harmonic $)\left[\mathrm{A}_{\mathrm{rms}}\right]$ & - & - & - & $63 \angle 76^{\circ}$ \\
\hline$I_{a}\left(7^{r d}\right.$ harmonic $)\left[\mathrm{A}_{\mathrm{rms}}\right]$ & - & - & - & $33 \angle-2^{\circ}$ \\
\hline
\end{tabular}

Table 4: Summary of Simulated Cases

\begin{tabular}{|c|c|c|c|c|c|}
\hline \multirow[t]{2}{*}{ Case } & \multicolumn{2}{|c|}{ Source } & \multicolumn{3}{|c|}{ Disturbing load } \\
\hline & Switch S-1 & Switch S-2 & Switch S-3 & Switch S-4 & Switch S-5 \\
\hline $\bar{A}$ & on & off & on & off & off \\
\hline $\boldsymbol{B}$ & on & off & off & on & off \\
\hline$C$ & on & off & off & off & on \\
\hline$D$ & on & off & on & on & on \\
\hline $\boldsymbol{E}$ & off & on & on & off & off \\
\hline $\boldsymbol{F}$ & off & on & off & on & off \\
\hline $\boldsymbol{G}$ & off & on & off & off & on \\
\hline $\boldsymbol{H}$ & off & on & on & on & on \\
\hline
\end{tabular}
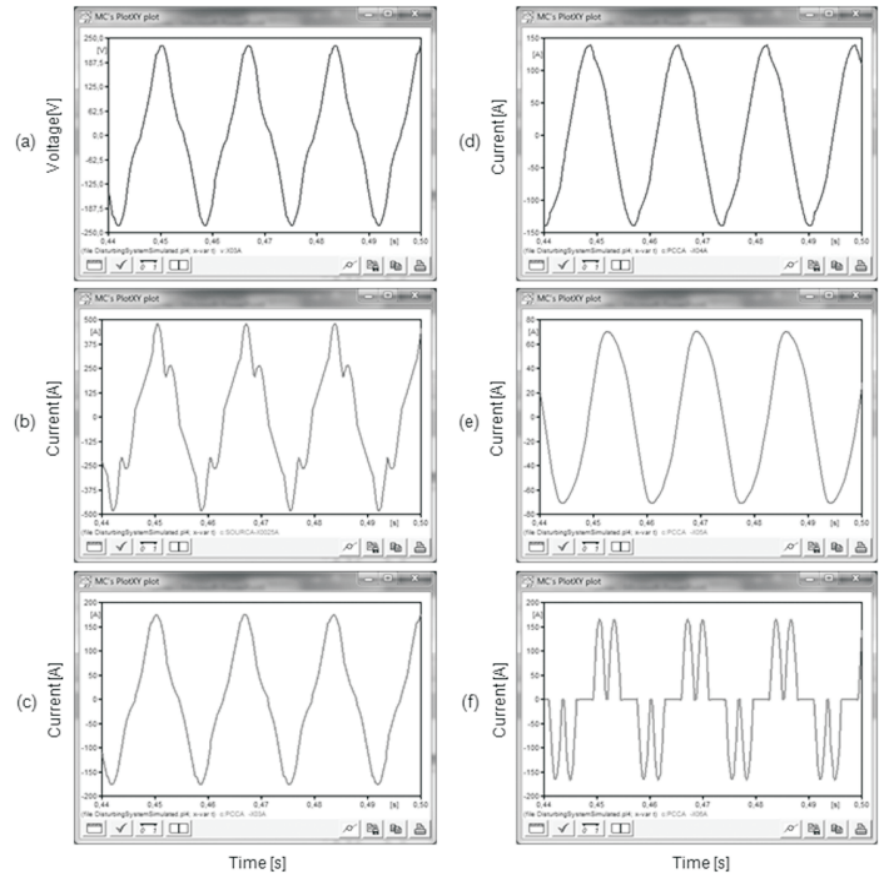

Figure 10.Voltage and current waveform for the case H: (a) PCC voltage, (b) PCC current, (c) ideal load current, (d) unbalanced load current (e) inductive load current and (f) nonlinear load current. 
Table 5: Summary of Simulation Results: Individual Indices

\begin{tabular}{|c|c|c|c|c|c|c|}
\hline \multirow[t]{2}{*}{ Case } & \multicolumn{3}{|c|}{$\overline{P C C}$} & \multicolumn{3}{|c|}{ Disturbing load } \\
\hline & $T H D_{I}[\%]$ & $U F[\%]$ & $Q F[\%]$ & $T H D_{I}[\%]$ & $U F[\%]$ & $Q F[\%]$ \\
\hline $\bar{A}$ & 0.23 & 33.08 & 0.00 & 0.23 & 100.00 & 0.00 \\
\hline$B$ & 0.90 & 0.25 & 32.93 & 0.89 & 0.25 & 81.52 \\
\hline$C$ & 19.80 & 0.24 & 0.01 & 59.73 & 0.22 & 0.03 \\
\hline$D$ & 12.52 & 17.93 & 20.75 & 21.92 & 27.93 & 33.25 \\
\hline$E$ & 21.06 & 31.71 & 0.00 & 3.45 & 100.00 & 0.00 \\
\hline $\boldsymbol{F}$ & 14.52 & 3.17 & 32.07 & 10.20 & 3.03 & 80.74 \\
\hline$G$ & 18.34 & 2.95 & -10.29 & 62.35 & 3.38 & -37.80 \\
\hline $\boldsymbol{H}$ & 15.72 & 17.62 & 15.81 & 28.32 & 29.10 & 26.37 \\
\hline
\end{tabular}

These results allow calculating of the global index GI.

Table 6 shows a comparative summary between the GI index and the $D F_{p q}$ factor for each of the cases. From the comparisons it can be said that:

- The global GI index and the $D F_{p q}$ deviation factor are very similar when the power supply is sinusoidal balanced (ideal supply) and the load connected to $\mathrm{PCC}$ is only unbalanced, inductive or nonlinear (case: $A, B$ and $C$ ).

- There are differences between the $D F_{p q}$ deviation factor and the GI global index when the power supply is not sinusoidal (case: $E, F$ and $G$ ). These differences are increased when different disturbing loads are connected simultaneously to the PCC (case: $D$ and $H$ ).

- The $D F_{p q}$ deviation factor provides a better alternative for the evaluation of a non-conformity regarding the electrical systems ideal condition. This assertion is based on the fact that $D F_{p q}$ evaluates more rigorously the loss of power quality when multiple disturbances affect simultaneously an electrical network, regardless of whether the disturbance arises from the load or from source.

\section{Conclusion}

The introduced mathematical tool proposes a way to calculate the instantaneous power components and the current decomposition in an active and a reactive component, based on a formal definition of reactive power in multiphase electric systems. This new formulation also allows the definition of instant compensation objectives in $n$-phase systems, based on the fact that the expression for the instantaneous reactive power in multiphase systems is known thanks to the orthogonal decomposition of the power tensor. This new approach allows the reduction of construction

Table 6: Summary of Simulation Results: Global Indices

\begin{tabular}{|c|c|c|c|c|}
\hline \multirow[t]{2}{*}{ Case } & \multicolumn{2}{|c|}{ PCC } & \multicolumn{2}{|c|}{ Disturbing load } \\
\hline & $G I[\%]$ & $F D_{p q}[\%]$ & $G I[\%]$ & $F D_{p q}[\%]$ \\
\hline $\bar{A}$ & 33.08 & 33.07 & 100.00 & 100.00 \\
\hline$B$ & 32.93 & 31.90 & 81.52 & 80.48 \\
\hline$C$ & 19.80 & 19.57 & 59.73 & 59.38 \\
\hline$D$ & 20.75 & 29.16 & 33.25 & 47.11 \\
\hline$E$ & 31.71 & 40.13 & 100.00 & 105.70 \\
\hline$F$ & 32.07 & 40.19 & 80.74 & 84.65 \\
\hline $\boldsymbol{G}$ & 18.34 & 28.41 & 62.35 & 82.66 \\
\hline $\boldsymbol{H}$ & 17.62 & 32.53 & 29.10 & 49.02 \\
\hline
\end{tabular}


costs of the APFs, given that now APFs can be designed at low voltages in the case of rectifiers of twelve, eighteen, twenty four and more pulses. A single $D F_{p q}$ index has been proposed as the basis of the power quality assessment based on the instantaneous power tensor theory. The deviation indicator of power quality proved to be successful, as found by comparisons made between the ideal power system introduced and many other typical loads selected from a variety of test cases. So, the New Theoretical Approach does not present any problem to be used as the base of an active power filter control algorithm or a global index for power quality assessment, with any voltage conditions in the point of common coupling (PCC). The results have been corroborated by the correspondent simulations in the Matlab-Simulink and ATPEMTP environments.

\section{List of Symbols}

\begin{tabular}{|c|c|}
\hline$\langle\cdot\rangle$ & scalar product \\
\hline$\times$ & vector product \\
\hline$\otimes$ & tensor product \\
\hline$\|\cdot\|$ & norm \\
\hline $\operatorname{tr}(\cdot)$ & trace \\
\hline$(\cdot)^{T}$ & transpose \\
\hline$\vec{v}$ & instantaneous voltage vector \\
\hline$\vec{i}$ & instantaneous eurrent vector \\
\hline$p$ & instantaneous active power \\
\hline$q$ & instantaneous reactive power \\
\hline$g_{i j}$ & instantaneous conductance tensor \\
\hline$b_{i j}$ & instantaneous suseeptance tensor \\
\hline$\wp$ & instantaneous power tensor \\
\hline$G_{e_{-} i j}$ & equivalent conductance tensor \\
\hline$B_{e_{-} i j}$ & equivalent susceptance tensor \\
\hline${ }^{i d e a l} \wp_{i j}$ & ideal power tensor \\
\hline$D F_{p q}$ & deviator factor of the power quality \\
\hline
\end{tabular}

\section{References}

Akagi, H., Kanazawa, Y., \& Nabae, A. (1984). Instantaneous reactive power compensators comprising switching devices without energy storage components. IEEE Transaction on Industry Applications 20(3), 625-631.
Cristaldi L., Ferrero, A., \& Salicone, S. (2002). A Distributed System for Electric Power Quality Measurement. IEEE Transactions on Instrumentation and Measurement 51(4), 776-778.

Czarnecki, L. S. (2004). On Some Misinterpretations of the Instantaneous Reactive Power p-q Theory. IEEE Transaction on Power Electronics 19(3), 828-836.

Dai, X., Liu, G., \& Gretsch, R. (2004). Generalized Theory of Instantaneous Reactive Quantity for Multiphase Power System. IEEE Transaction on Power Delivery 19(3), 965-972.

Dell'Aquila, A., Marinelli, M., Monopoli, V. G., \& Zanchetta, P. (2004). New Power-Quality Assessment Criteria for Supply Under Unbalanced and Nonsinusoidal Conditions. IEEE Transaction on Power Delivery 19(3), 1284-1290.

Depenbrock, M., Staudt, V., \& Wreder, H. (2003). A theoretical investigation of original and modified instantaneous power theory applied to four-wire systems. IEEE Transaction on Industry Applications 39(4), 1160-1167.

Depenbrock, M. (1993). The FBD-Method, A Generally Applicable tool for Analyzing Power Relations. IEEE Transactions on Power Systems 8(2), 381-387.

Emanuel, A.E. (2004). Summary of IEEE Standard 1459: Definitions for the Measurement of Electric Power Quantities Under Sinusoidal, Nonsinusoidal, Balanced, or Unbalanced Conditions. IEEE Transaction on Industry Applications 40(3), 869-876.

Ferrero, A. (2008). Measuring Electric Power Quality: Problems and Perspectives. Measurement 41(2), 121-129.

Itskov, M. (2007). Tensor Algebra and Tensor Analysis for Engineers: with Applications to Continuum Mechanics, Springer, Berlin.

Leon, F. de, \& Cohen, J. (2008). Discussion of Instantaneous Reactive Power p-q Theory and Power Properties of Three-Phase Systems. IEEE Transaction on Power Delivery 23(3), 1693-1694. 
Lin, T., \& Domijan, A. (2005). On power quality indices and real time measurement. IEEE Transaction on Power Delivery 20(4), 25522562.

Montero, M.I.M., Cadaval, E.R., \& Gonzalez, F.B. (2007). Comparison of Control Strategies for Shunt Active Power Filters in Three-Phase FourWire Systems. IEEE Transaction on Power Electronics 22(1), 229236.

Morsi, W.G., \& El-Hawary M.E. (2007). Defining power components in nonsinusoidal unbalanced polyphase systems: the issues. IEEE Transaction on Power Delivery 22(4), 24282438.

Salmerón, P., \& Herrera, R.S. (2009). Instantaneous Reactive Power Theory A General Approach to Poly-Phase Systems. Electric Power Systems Research. 79, 1263-1270.

Salmerón, P., \& Litran, S.P. (2010). Improvement of the Electric Power Quality Using Series Active and Shunt Passive Filters. IEEE Transaction on Power Delivery 25(2), 1058-1067.

Shin, Y.J., Powers, E.J., Grady, M. \& Arapostathis, A. (2006). Power Quality Indices for Transient Disturbances. IEEE Transaction on Power Delivery 21(1), 253-261.

Ustariz, A.J. Cano, E.A. and Tacca, H.E. (2010). Instantaneous Power Tensor Theory: Improvement and Assessment of the Electric Power Quality. In Proceedings of the 14th International Conference on Harmonics and Quality of Power (ICHQP), Bergamo, Italy, pp. 1-

Willems, J.L. (1992). A New Interpretation of the Akagi-Nabae Power Components for Nonsinusoidal Three-phase Situations. IEEE Transactions on Instrumentation and Measurement 41(4), 523-527. 